\title{
Broad overview of oxidative stress and its complications in human health
}

\author{
Peter Kovacic $^{1^{*}}$, Ratnasamy Somanathan ${ }^{1,2}$ \\ ${ }^{1}$ Department of Chemistry and Biochemistry, San Diego State University, San Diego, USA; \\ *Corresponding Author: pkovacic@mail.sdsu.edu \\ ${ }^{2}$ Centro de Graduados e Investigación del Instituto Tecnológico de Tijuana, Tijuana, Mexico
}

Received 2 November 2012; revised 5 December 2012; accepted 14 December 2012

\begin{abstract}
There is extensive literature dealing with toxicity and human health. A goodly portion puts focus on involvement of electron transfer, reactive oxygen species and oxidative stress involving body organs. There is evidence for prevention or amelioration by antioxidants. This is one mechanism which is part of a multifaceted mode of action. This review comprises an update of earlier literature.
\end{abstract}

Keywords: Health; Organ Toxicity; Oxidative Stress; Reactive Oxygen Species; Electron Transfer

\section{INTRODUCTION}

Abundant literature exists on involvement of reactive oxygen species (ROS) and oxidative stress (OS) on human health. Various reviews (see main text) in the past have addressed the topic in relation to organ toxicity. The present review provides a literature update including the following organs: lungs, skin, kidney, heart, reproductive and mitochondria. The preponderance of bioactive substances or their metabolites incorporate electron transfer (ET) functionalities, which, we believe, play an important role in physiological responses. These main groups include quinones (or phenolic precursors), metal complexes (or complexors), aromatic nitro compounds (or reduced hydroxylamine and nitroso derivatives), and conjugated imines (or iminium species). In vivo redox cycling with oxygen can occur giving rise to OS through generation of ROS (Scheme 1), such as hydrogen perox- ide, hydroperoxides, alkyl peroxides, and diverse radicals (hydroxyl, alkoxyl, hydroperoxyl, and superoxide). In some cases, ET results in interference with normal electrical effects, e.g., in respiration or neurochemistry. Generally, active entities possessing ET groups display reduction potentials in the physiologically responsive range, i.e., more positive than $-0.5 \mathrm{~V}$. ET, ROS, and OS have been increasingly implicated in the mode of action of drugs and toxins (toxicants), e.g. anti-infective agents [1], anticancer drugs [2,3], carcinogens [4], and toxins [5].

There is a plethora of experimental evidence supporting the theoretical framework, including generation of the common ROS, lipid peroxidation, degeneration products of oxidation, depletion of AOs, effect of exogenous AOs, DNA oxidation and cleavage products, as well as electrochemical data. This comprehensive, unifying mechanism is in keeping with the frequent observations that many ET substances display a variety of activities, e.g., multiple drug properties, as well as toxic effects.

Diverse mechanisms have been proposed for these agents. However, there has not been recognition for a unifying theme involving ET-ROS-OS. The unifying relationships lend credence to the proposed involvement of ET-ROS-OS in the physiological effects addressed in this review, and comprise an extension of the prior mechanistic framework. However, it should be emphasized that physiological activity is often complex and multifaceted, with various modes of action involved. A number of original references may be found in the reviews and articles cited; in many cases, references are representative.

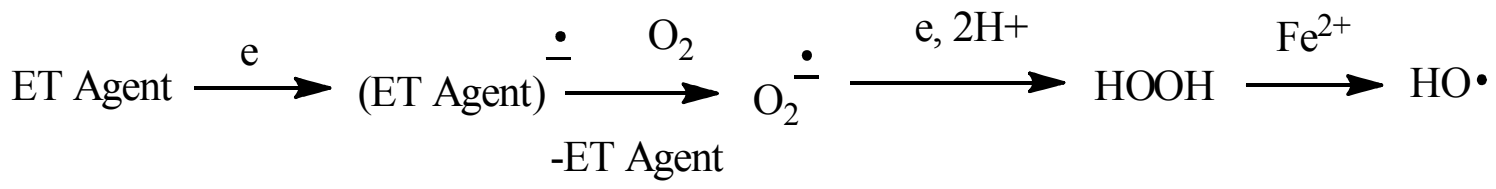

Scheme 1. Formation of ROS and RNS. Shown is ET resulting in formation of superoxide which serves as precursor of other ROS. 


\section{DISCUSSION}

\subsection{Neurodegenerative Diseases}

There has been treatment of neurotoxicity and neurodegenerative diseases involving ROS and OS. A recent review represents an update of neurodegenerative diseases based on extensive literature [6]. The redox approach comprises a unifying theme which can be applied to a large number of illnesses in this class, including Parkinson's, Huntington's, Alzheimer's, prions, Down's syndrome, ataxia, multiple sclerosis, Creutzfeldt-Jacob disease, amyotrophic lateral sclerosis, schizophrenia, and tardive dyskinesia. An earlier review addressed neurodegeneration from a similar mechanistic viewpoint based on ROS-OS [7].

The brain consumes more oxygen under physiological conditions than other organs, thereby increasing its susceptibility to OS since generation of higher levels of ROS can lead to pathological changes when these are in excess of the buffering capacity of endogenous antioxidant systems [8]. Extensive data on OS, signaling pathways, cell death and neuroprotection have been generated in many studies.

Hyperoxia produces toxicity, including that of the nervous system. The mammalian brain appears to be particularly sensitive to oxidative damage, one reason being the high oxygen consumption. Rises in calcium interfere with mitochondrial function (including neural), increasing formation of superoxide which can react with nitric oxide (NO) to form the potent oxidant peroxynitrite $\left(\mathrm{ONOO}^{-}\right)$, accompanied by lipid peroxidation. Several neurotransmitters, including dopamine, L-DOPA, serotonin and norepinephrine, can produce ROS, evidently via quinone/semiquinone metabolites. Iron is found throughout the brain as complexes with various proteins (see Role of Iron below). Neural membrane lipids are replete with polyunsaturated fatty acids whose oxidation products, such as 4-hydroxynonenal and 4oxononenal [9], can act as sources of ROS, and are especially cytotoxic to neurons. Brain metabolism generates an abundance of hydrogen peroxide via SODs (superoxide dismutases) and other enzymes [7]. AO defenses are modest, such as catalase levels. Brain microglia can become activated to produce superoxide, hydrogen peroxide and cytokines. Microglia and astrocytes are major players in brain inflammation which is associated with ROS [10]. Some cytochromes leak electrons during the catalytic redox cycle, thus providing ROS [7]. An- other source of brain ROS is NADPH oxidase enzymes. Hemoglobin, a neurotoxin, can release heme which is a powerful promoter of lipid peroxidation. The complex of hemoglobin with NO can also generate OS [11]. The Halliwell review presents various means for defense against neurotoxins [7]. There is also reference to earlier treatment of neurodegenerative diseases.

A broad overview of neurotoxins was presented based on electron transfer (ET), reactive oxygen species (ROS), and oxidative stress (OS) [12]. It is relevant that metabolites from toxins generally posses ET functionalities which can participate in redox cycling. Toxic effects at the molecular level include lipid peroxidation, DNA attack, adduction, enzyme inhibition, oxidative attack on the CNS, and cell signaling. The toxins fall into many categories. Beneficial effects of AOs are documented. A related update was reported in 2012 [13]. A similar article deals with nitric oxide (NO), catecholamine and glutamate [14]. The review treats the mechanism of these agents as important neurotransmitters and as neurotoxins, based on involvement of ET-ROS-OS.

\section{Role of Iron}

A recent review presents a unifying theme for cellular death and neurotoxicity by iron agents $[6,15]$. The basic theme involves continuing and autocatalytic generation of hydroxyl radicals by way of the Fenton reaction involving poorly liganded iron.

\section{PULMONARY TOXICITY}

The pulmonary system is one of the main targets for toxicity. In the industrial age, there has been a large increase in atmospheric pollutants. Many adverse reactions can occur, some of the principal ones being asthma, COPD and cancer.

It is often unclear what role natural components play in the mechanism of pathogenesis. However, a common factor appears to be the upregulation of ROS in lung cells upon exposure. In vivo redox cycling with oxygen can occur giving rise to OS through generation of ROS. ROS can arise from diverse sources, both endogenous and exogenous [16]. Reduction of $\mathrm{O}_{2}$ to ROS, e.g., superoxide, occurs as a by-product of metabolism. When cellular injury occurs, release of species, such as iron, into extracellular space can lead to generation of deleterious ROS. Neutrophils and macrophages are adept at transforming oxygen into ROS which eliminate foreign organisms, accompanied by the undesirable effect of OS on normal cells. The lung is especially susceptible to injury by this gas. For example, hyperoxia damages endothelial and alveolar epithelial cells [17]. A recent review deals with the consequence of hyperoxia and toxicity of oxygen in the lung [18]. Exposure results in increased intracellular generation of superoxide and, hence, of other ROS, with ensuing autoxidation reactions.

A dramatic example of lung toxicity involving ROS is adult respiratory distress syndrome (ARDS) which is brought about by trauma, shock, sepsis, vomiting, and inhalation of toxins. Inflammation, a common result of 
lung insult by toxic substances, is a precursor of subsequent events triggered by ROS. There is considerable evidence that OS is a contributing factor in ARDS [17]. We wish to emphasize the following quote: "In general, free radicals represent an important component in the pathogenesis of lung disease" [16]. The role of ROS in lung damage is further buttressed by the increased activeity of free radical-scavenging enzymes in lungs challenged by a variety of toxins.

There is appreciable literature on ROS, OS and pulmonary toxicity. In our 2009 review, we surveyed a number of pulmonary toxicants [16]. In this review only those toxicants from more recent years will be cited.

\subsection{Role of Nanomaterials}

In recent years, a wide range of nanomaterials have been developed for various applications. Increasing evidence suggests that the special physicochemical properties of these nanomaterials pose potential risk to human health. Recent reviews in this area deal with the biomechanism and toxicity of nanoparticles, including pulmonary insults $[19,20]$.

Data indicate that the composition and size of nanomaterials, as well as the target cell type, are critical determinants of intracellular responses, degree of cytotoxicity and potential mechanism of toxicity [21]. ROS plays a major role in its toxicity. Alveolar epithelial cells exposed to manganese (II,III)oxide nanoparticles generated ROS leading to OS and apoptosis [22]. Copper oxide nanoparticles induce OS and cytotoxicity in airway epithelial cells [23].

A study correlated the conduction band energy with cellular redox potential of $\mathrm{Co}_{3} \mathrm{O}_{4}, \mathrm{Cr}_{2} \mathrm{O}_{3}, \mathrm{Ni}_{2} \mathrm{O}_{3}, \mathrm{Mn}_{2} \mathrm{O}_{3}$ and $\mathrm{CoO}$ nanoparticles and its ability to induce oxygen radicals, OS, and inflammation [24]. A review deals with the mammalian toxicity of $\mathrm{ZnO}$ nanoparticles, through inhalation results in ROS generation which plays an important role in inflammatory response [25]. A 2012 review covers toxicity induced by nanoparticles [26]. Multiwalled carbon nanotubes induce OS, with increased ROS production and depletion of intracellular GSH. Multiwalled carbon nanotubes also induce a fibrogenic response by stimulating ROS production [27]. Data showed that exposure of cultured RAW 264.7 cells and A549 human lung cells to multiwalled carbon nanotubes led to OS induced cytotoxicity [28].

\subsection{Role of Organic Toxins}

OS and oxidative effects on DNA are increased in mice exposed to styrene or styrene oxide, and these may play a role in the lung tumorigenesis [29]. In a related study $\mathrm{N}$-acetylcysteine and GSH were shown to act as AOs in preventing OS in mice exposed to styrene [30].
Chlorobenzene and 1,2-dichlorobenzene cause OS and induce apoptosis in lung epithelial cells at non-acute toxic concentrations [31]. 2-Chloroethyl ethyl sulfide, a sulfur mustard, causes a significant increase in mitochondrial dysfunction, involving increase in ROS in lung cell injury [32]. Metalloporphyrin acts as an AO in decreasing mitochondrial ROS, DNA oxidation and the increasing intracellular GSH. A study revealed diallyl trisulfide, a major constituent of garlic oil, induces apoptosis of U937 human leukemia cells by generation of ROS [33].

Research showed that wood dust from pine, birch and oak is cytotoxic, being able to increase the production of ROS [34].

\section{DERMAL TOXICITY}

Insults to the skin may be mild, serious or lethal. Various constituents of the skin may be affected by dermal toxicants. Cutaneous damage may also result from inhalation or ingestion of toxins, in addition to direct skin contact. Similarly, substances that induce toxicity through absorption by the skin can also migrate to and adversely affect other organs.

In this review, we draw lines of evidence to support the concept that the ET-ROS-OS unifying theme, which has been successful in describing the means by which many other classes of toxins induce their effects, can also be applied to dermatotoxins [35]. Such toxin classes include a variety of structurally diverse substances.

Exposure to the chemical warfare agent sulfur mustard is reported to cause depletion of GSH, which plays an important role in sulfur mustard-linked OS and skin injury. Cultured skin epidermal cells and SKH-1 mouse skin when exposed to 2-chloroethyl ethyl sulfide, an analog of mustard gas, led to amelioration by GSH and induction of toxicity [36]. N-Acetyl cysteine, a GSH analog, acts as an $\mathrm{AO}$ and shows both protective and therapeutic effects. The sulfur mustard analog 2chloethyl ethyl sulfide induced oxidative DNA damage in skin epidermal cells and fibroblasts [37]. A related study also revealed that the sulfur mustard analog induces OS and activates transcription factors AP-1 and $\mathrm{NF}-\kappa \mathrm{B}$ via upstream signaling pathways including MAPKs and Akt in SKH-I hairless mouse skin [38].

$\mathrm{Cr}(\mathrm{IV})$ induced apoptosis with involvement of reactive oxidants [39]. Data indicated that topical exposure to unpurified single walled carbon nanotubes induced free radical generation, OS, and inflammation, depletion of glutathione, oxidation of protein thiols and carbonyls, elevated myeloperoxidase activity and skin thickening [40]. Amorphous nanosilica induces endocytosis-dependent ROS generation and DNA damage in human keratinocytes [41]. Cytotoxicity of uranium, has been in the spotlight in recent decades. Uranyl acetate induces 
OS [42].

Carotenoids are known to be potent quenchers of singlet molecular oxygen. Solar light-induced photooxidative stress causes skin photoaging by accelerating the generation of ROS. Intake of carotene lowered the metalloproteinase family which is responsible for wrinkles and sagging of skin, and helps in slowing down ultraviolet-A-induced photoaging in human skin by acting as a singlet oxygen quencher [43]. A 2012 review deals with protective mechanisms of green tea AOs polyphenols in skin [44]. Another article deals with the ROS-mediated skin damage and skin protection by AOs [45].

\section{NEPHROTOXICITY}

Toxic processes have been implicated in the pathogenesis of several systemic diseases including kidney, which induces OS in the kidney [46]. A 2012 review summarizes the induction of OS in kidney [46]. Much of the nephrotoxicity can be attributed to OS-induced by drugs, and reports address the role of prescription druginduced OS and toxicity $[47,48]$.

Drug-induced OS is implicated as a mechanism of toxicity in numerous organs, including liver. Well characterized drugs associated with OS, include cancer therapies, anti-inflammatory drugs, antiviral agents, antipsychotics and analgesics [48]. Metabolism of a drug may generate a reactive intermediate that can induce ROS generation, leading to OS in various organs. Cisplatin is an example of a drug that exhibits multi organ toxicity, acting as an antineoplastic agent. Clinically, renal injury has been described. The kidney accumulates cisplatin to a greater degree than other organs [48]. The disproportionate accumulation of cisplatin in kidney tissue contributes to free radical generation, depletion of antioxidants leading to OS and nephrotoxicity $[48,49]$. Research also indicated that cisplatin nephrotoxicity is associated with mutual mitochondrial/lysosomal potentiation (cross talk) of OS in renal proximal tubular cells [50]. This cross-talk results in release of lysosomal digestive protease and phospholipases and mitochondrial permeability transition pore opening leading to cytochrome c release and activation of caspase cascade, which signals apoptosis.

Several reports exist for AO action against cisplatin nephrotoxicity. Selenium nanoparticles functionalized with 11mercapto-1-undecanol inhibit ROS-mediated apoptosis [51]. Captopril, an angiotensin-converting enzyme inhibitor containing the sufhydryl group can protect against cisplatin-induced nephrotoxicity in rats [52]. Canabidiol attenuates cisplatin-induced nephrotoxicity by decreasing OS, inflammation and cell death [53].

Data demonstrate a key role of lysosomal iron and early ROS production in gentiomicin-induced lysosomal membrane premeabilization and apoptosis [54]. Salicylic acid was found to attenuate the nephrotoxicity in rats [55]. Ifosfamide, an antineoplastic drug, causes severe nephrotoxicity. The metabolite chloroactaldehyde is believed to be the chemical responsible for the nephrotoxicity [56]. N-Acetyl cysteine acts as an AO in decreasing the nephrotoxicity. A study showed that ROS, OS, and MAPK signaling is involved in promoting cyclosporineinduced glomerular dysfunction and subsequent nephrotoxicity [57].

Increased production of ROS by anticancer drugs trichostatin and 5-aza-deoxycytidine, has been described in patients with various malignancies, which is attributed to their nephrotoxicity.

A study showed uric acid attenuates toxicity by preventing systemic and renal oxidative stress and tissue damage induced by mercuric chloride in rats [58]. Cadmium $(\mathrm{Cd})$ is a well known human carcinogen and potent nephrotoxin. $\mathrm{Cd}$ caused renal toxicity by inducing lipid peroxidation and morphological alterations [59]. Curcumin, a natural product from turmeric protects $\mathrm{Cd}$-induced nephrotoxicity in rats. Colistin (polymyxin E), a cationic polypeptide antibiotic, causes OS-induced nephrotoxicity. Melatonin was found to attenuate colistin-induced nephrotoxicity in rats [60]. Zinc oxide and cadmium sulfide nanoparticles generate ROS that leads to OS-induced nephrotoxicity [61].

\section{CARDIOVASCULAR TOXICITY}

As described in drug-induced OS and nephrotoxicity, many antibiotic drugs also induce cardiotoxicity $[48,62]$. The antineoplastic activity of doxorubicin is mediated by interaction with DNA [48]. Reduction of doxorubicin by one electron via mitochondrial reductase may generate anthracycline semiquinone free radicals that produce ROS [48]. Reaction between iron and doxorubicin may also generate ROS. Extensive data have been generated in numerous model systems showing that administration of AOs protects cardiomycetes from doxorubicin-induced damage. The range of molecules explored is diverse, including plant extracts, vitamins $\mathrm{C}$ and $\mathrm{E}, \mathrm{N}$ acetylcycteine, L-carnitine, beta-blocker carvedilol, coenzyme Q10, and dexrazonxane $[48,63]$.

Adriamycin is another antineoplastic anthracycline used to treat solid tumors and various forms of cancer, but it displays cardiac toxicity [64]. Adriamycin is well known to produce large amounts of ROS, which may be lethal to cancerous cells. However, unchecked ROS generation typically leads to OS. Adriamycin has been documented to cause oxidative damage in several organs, including heart [64]. Gamma-glutamycysteine ethyl ester was found to suppress the OS induced by adriamycin. Several reviews deal with the mechanism and protection 
in anthracycline-induced cardiotoxicity [65-68].

Platinum-based compounds are commonly used cytotoxic agents in the treatment of several solid tumors. However, their application is still limited in elderly patients, due to the risk in cardiovascular toxicity. The increased risk is mainly due to ROS production [69]. AOs have been involved in cancer treatment by their property to suppress the oxidant injury $[69,70]$.

Administration of glucose degradation products increased cardiovascular damage in rat models [71]. A related study showed that glucose degradation products and advanced glycation end (AGEs) products play a role in the pathophysiolgy of cardiotoxicity [72]. Mechanistic aspects of AGEs have been addressed [73].

Cocaine is one of the most common illicitly used drugs in the world and causes the most frequent drugrelated deaths in young adults. Chronic cocaine consumption is associated with serious cardiovascular complications. Chronic cocaine administration causes severe myocardial OS through increased ROS production [74]. Cocaine cardiotoxicity may be mediated indirectly through its sympathomimetic effect, inhibiting reuptake and increasing the levels of neuronal catecholamines.

Exposure to nanoparticles significantly impairs endothelium-dependent vasoreactivity in coronary arterioles, and this may be due in large part to increases in microvascular ROS [75]. Another study showed that exposure to ambient particulate pollution induces arrhythmia via OS and calcium calmodulin kinase II activation [76].

In recent years two reviews have appeared dealing with heavy metal $(\mathrm{Ar}, \mathrm{Pb}, \mathrm{Cd}, \mathrm{Hg}$ ) poisoning and cardiovascular disease. The reviews implicate ROS and OS as major players in the pathophysiology of atherosclerosis $[77,78]$.

\section{REPRODUCTIVE TOXICITY}

Several earlier reviews have addressed this topic [7981]. The present contribution provides recent developments. There is evidence that several teratogens affect the developing embryo by increasing OS resulting in severe embryonic damage. This mechanism seems to operate in diabetic-induced embryonic damage, as well as in the mechanism of teratogenicity caused by ionizing radiation, hypoxia, alcohol and cocaine use and cigarette smoking. Under diabetic conditions, there was a significant decrease in the activity of endogenous $\mathrm{AO}$ enzymes and of vitamins $\mathrm{C}$ and $\mathrm{E}$ in the embryos. Human and animal studies show that the main mechanism of fetal damage induced by high levels of ionizing irradiation, cocaine and alcohol abuse, hypoxia and cigarette smoking is also by increased embryonic OS. Similarly, several drugs exert their teratogenic activity via embryonic OS. Abnormal placentation may also cause enhanced placental OS, resulting in embryonic death. Animal studies also show that a variety of AOs are effective in decreasing the damaging effects of hightened OS induced by teratogens. Concurrent administration of chloroamphenicol (CAP) with multivitamin-haematinics complex (MHC) is a common practice to cushion anticipated anaemic effects in reproductive toxicity [82]. Alone, MHC treatment markedly decreased catalase (CAT) and glutathione S-transferase (GST) activities, whereas it resulted in significant increase in superoxide dismutase (SOD) activity. Significant increase in testicular lipid peroxidation and sperm abnormalities were accompanied by reduction in sperm number, sperm motility and live-dead ratio in all treatment groups. MHC-induced testicular toxicity occurred vis OS.

Mequindox (MEQ) is a synthetic antimicrobial chemical [83]. A study was designed to investigate the hypothesis that MEQ exerts testicular toxicity by causing OS. Superoxide dismutase (SOD), reduced glutathione (GSH) and 8-hydroxydeoxyguananosine (8-OHdG) levels were elevated, whereas the malondialdehyde (MDA) level was slightly increased. The findings provide evidence in vivo for the formation of free radicals.

Reports document the protective effect of AOs, such as vitamin E [84] and Ginko biloba [85]. Various studies on reproductive toxicity deal with the role of metals: $\mathrm{Pb}$ [86], Cr [87,88], and Se [89].

\section{MITOCHONDRIA}

Mitochondrial OS has long been implicated in normal aging, and a host of human diseases, including cancer and neurodegenerative disorders. Mitochondria are the major source of oxygen free radicals in most cell types. Low concentrations of ROS can serve as signaling functions, triggering the activation of specific pathways [90]. However, high concentrations of ROS cause lipid peroxidation, damage to cell membranes, proteins, and DNA. Mitochondria are, therefore, a major source of ROS and a major target of ROS-induced OS and damage. A high rate of mitochondrial DNA mutation eventually exacerbates mitochondrial dysfunction and reduces mitochondrial energy production [90].

Under OS conditions, mitochondria release various pro-apoptoic factors. This release is caused by the permeabilization of the mitochondrial outer membrane, which accompanies the depolarization of the mitochondrial intermembrane potential. Aldehydes are generated during numerous physiological processes. Aldehydes are highly reactive agents, which form adducts with lipids, proteins, and DNA, affecting the function of these macromolecules. Aldehyde dehydrogenases are important enzymes that eliminate toxic aldehydes by catalyzing their oxidation to non-reactive acids. A review discusses the mitochobdrial aldehyde dehydrogenase and cardiac diseases [91]. Inhibition of aldehyde dehydrogenase 2 by 
OS is associated with cardiac dysfunction in diabetic rats [92]. A study demonstrated that TNF- $\alpha$-induced OS alters redox homeostasis by impairing the membrane permeability transition pore opening proteins adenine nucleotide translocator and voltage-dependent anion channel, thereby resulting in the pore opening, causing mitochondrial dysfunction and attenuated cardiac function [93].

A recent review deals with the mitochondria death/ survival signaling pathways in cardiotoxicity induced by anthracyclines [94]. Data shows that inhibition of mitochondrial transition permeability prevents doxorubicininduced cardiotoxicity. OS and mitochondrial dysfunction have been implicated in atherosclerosis [95]. Authors suggest mitochondria-targeted AOs as potential therapy. Alzheimer's disease, the most common form of dementia with a progressive course, evidences neuronal damage in specific vulnerable brain regions and circuits involved in memory and language [96]. Two recent reviews deal with mitochondrial- and endoplasmic reticulum-associated OS in Alzheimer's disease [96,97].

There is an emerging consensus that aging is a multifactorial process, which is genetically determined and influenced epigenetically by environment. OS induced DNA damage, oxidation of proteins, lipid peroxidation and ROS have been implicated as causative factors of aging [98]. Two reviews deal with the OS, mitochondrial dysfunction and aging [98,99].

\section{CONCLUSION}

This review presents recent reports dealing with electron transfer, reactive oxygen species and oxidative stress as part of the mechanism of toxicity involving organs in human health. Various portions of the organ cells may be involved in the insults. Antioxidants may play a role in prevention.

\section{ACKNOWLEDGMENTS}

Editorial assistance by Thelma Chavez is acknowledged.

\section{REFERENCES}

[1] Kovacic, P. and Becvar L.E. (2000) Mode of action of anti-infective agents: Emphasis on oxidative stress and electron transfer. Current Pharmaceutical Design, 6, 143167. doi:10.2174/1381612810006020143

[2] Kovacic, P. and Osuna J.A. (2000) Mechanisms of anticancer agents: Emphasis on oxidative stress and electron transfer. Current Pharmaceutical Design, 6, 277-309. doi:10.2174/1381612003401046

[3] Kovacic, P. (2007) Unifying mechanism for anticancer agents involving electron transfer and oxidative stress: Clinical implications. Medical Hypotheses, 69, 510-516. doi:10.1016/j.mehy.2006.08.046
[4] Kovacic, P. and Jacintho, J.D. (2001) Mechanism of carcinogenesis: Focus on oxidative stress and electron transfer. Current medicinal chemistry, 8, 773-796. doi:10.2174/0929867013373084

[5] Kovacic, P. and Somanathan, R. (2008) Unifying mechanism for eye toxicity: Electron transfer, reactive oxygen species, antioxidant benefits, cell signaling and cell membranes. Cell Membranes and Free Radical Research, 1, 56-69 (and references therein).

[6] Kovacic, P. and Somanathan, R. (2012) Redox process in neurodegerative disease involving reactive oxygen species. Current Neuropharmacology, 10, in Press.

[7] Halliwell, B. (2006) Oxidative stress and neurodegeneration: Where are we now? Journal of Neurochemistry, 97, 1634-1658. doi:10.1111/j.1471-4159.2006.03907.x

[8] Robert, R., Smith, R.A., Safe, S., Szabo, C., Tjalkens, R. B. and Robertson, F.M. (2010) Toxicological and pathphysiological roles of reactive oxygen and nitrogen species. Toxicology, 276, 85-94.

doi:10.1016/j.tox.2010.07.009

[9] Kovacic, P. (2006) Novel electrochemical approach to enhanced toxicitry of 4-oxo-2-nonenal vs. 4-hydroxy-2nonenal (role of imine): Oxidative stress and therapeutic modalities. Medical Hypotheses, 67, 151-156. doi:10.1016/j.mehy.2005.10.034

[10] Min, K.J., Yang, M.S., Kim, S.U. and Joe, E.H. (2006) Astrocytes induce hemeoxygenase-1 expression in microglia: A feasible mechanism for preventing excessive brain inflammation. The Journal of Neuroscience, 26, 18801887. doi:10.1523/JNEUROSCI.3696-05.2006

[11] Chiu, D.T., van den Berg, J., Kuypers, F.A., Hung, I.-J., Wei, J.-S. and Liu, T.-Z. (1996)Correlation of membrane lipid peroxidation with oxidation of hemoglobin variants: Possibly related to the rates of hemin release. Free Radical Biology \& Medicine, 21, 89-95. doi:10.1016/0891-5849(96)00035-4

[12] Kovacic, P. and Somanathan, R. (2012) Nervous about developments in electron transfer-ROS-oxidative stress: mechanisms of neurotoxicity? In: Laher, J, Ed., Systems Biology of Free Radicals and Antioxidants, Springer Verlag, New York, in Press.

[13] Kovacic, P. and Somanathan, R. (2005) Neurotoxicity: the broad framework of electron transfer, oxidative stress and protection by antioxidants. Current Medicinal Chemistry - Central Nervous System Agents, 5, 249-258. doi:10.2174/156801505774913044

[14] Jacintho, J.D. and Kovacic, P. (2003) Neurotransmission and neurotoxicity by nitric oxide, catecholamines, and glutamate: Unifying themes of reactive oxygen species and electron transfer. Current Medicinal Chemistry, 10, 2693-2703. doi:10.2174/0929867033456404

[15] Kell, D.B. (2010) Towards a unifying, systems biology understanding of large-scale cellular death and destruction caused by poorly liganded iron: Parkinson's, Huntington's, Alzheimer's. prions, bactericides, chemical toxicology and others as examples. Archives of Toxicology, 84, 825-889. doi:10.1007/s00204-010-0577-x

[16] Kovacic, P. and Somanathan, R. (2009) Pulmonary toxic- 
ity and environmental contamination: Radicals, electron transfer, and protection by antioxidants. Reviews of Environmental Contamination and Toxicology, 201, 41-69. doi:10.1007/978-1-4419-0032-6 2

[17] Halliwell, B. and Gutteridge, J.M.C. (1999) Free Radicals in Biology and Medicine. Oxford University Press, New York, 1-897.

[18] Mach, W.J., Thimmesch, A.R., Pierce, J.T. and Pierce, J.D. (2011) Consequence of hyperoxia and the toxicity of oxygen in the lung. Nursing Research and Practice, 2011, Article ID: 260482, 7 Pages. doi:10.1155/2011/260482

[19] Kovacic, P. and Somanathan, R. (2010) Biomechanisms of nanoparticles (toxicants, antioxidants and therapeutics): Electron transfer and reactive oxygen species. Journal of Nanoscience and Nanotechnology, 10, 1-12. doi:10.1166/jnn.2010.3028

[20] Jia'en Li, J., Muralikrishnan, S., Ng, C.-T., Yung, L.Y.L. and Bay, B.-H. (2010) Nanoparticle-induced pulmonary toxicity. Experimental Biology and Medicine, 235, 10251033. doi:10.1258/ebm.2010.010021

[21] Sohaebuddin, S.K., Thevenot, P., Baker, D., Eaton, J.W. and Tang, L. (2012) Nanomaterial cytotoxicity is composition, size, and cell type dependent. Particle and Fibre Toxicology, 7, 1-17.

[22] Frick, R., Müller-Edenborn, B., Sclicker, A., RothenRutishauser, B., Raemy, D.O., Günther, D., Hattendorf, B., Stark, W. and Beck-Schimmer, B. (2011) Comparison of manganese oxide nanoparticles and manganese sulfate with regard to oxidative stress, uptake and apoptosis in alveolar epithelial cells. Toxicology Letters, 205, 163-172. doi:10.1016/j.toxlet.2011.05.1037

[23] Fahmy, B. and Cormier, S.A. (2009) Copper oxide nanoparticles induce oxidative stress and cytotoxicity in airway epithelial cells. Toxicology in Vitro, 23, 1365-1371. doi:10.1016/j.tiv.2009.08.005

[24] Zhang, H., Ji, Z., Xia, T., Meng, H., Low-Kam, C., Liu, R., Pokhrel, S., Lin, S., Wang, X., Liao, Y.-P., Wang, M., Li, L., Rallo, R., Damoiseaux, R., Telesca, D., Mädler, L., Cohen, Y., Zink, J. I. and Nel, A.E. (2012) Use of metal oxide nanoparticle band gap to develop a predictive paradigm for oxidative stress and acute pulmonary inflammation. ACS Nano, 6, 4349-4368. doi:10.1021/nn3010087

[25] Vanderbriel, R.J. and De Jong, W.H. (2012) A review of mammalian toxicity of $\mathrm{ZnO}$ nanoparticles. Journal of Nanotechnology, Science and Applications, 5, 61-71. doi:10.2147/NSA.S23932

[26] Shinde S.K., Grampurohit, N.D., Gaikwad, D.D., Jadhav, S.L., Gadhave, M.V. and Shelke, P.K. (2012) Toxicity induced by nanoparticles. Asian Pacific Journal of Tropical Disease, 2, 331-334. doi:10.1016/S2222-1808(12)60072-3

[27] He, X., Young, S.H., Schwegler-Berry, D., Chisholm, W.P., Fernback, J.E. and Ma, Q. (2011) Multiwalled carbon nanotubes induce a fibrogenic response by stimulating reactive oxygen species production, activating NF$\kappa \mathrm{B}$ signaling, and promoting fibroblast-to-myofibroblast transformation. Chemical Research in Toxicology, 24, 22372248. doi:10.1021/tx200351d

[28] Chen, B., Liu, Y., Song, W.M., Hayashi, Y., Ding, X.C. and Li, W.H. (2011) In vitro evaluation of cytotoxicity and oxidative stress induced by multiwalled carbon nanotubes in murine RAW 264.7 macrophages and human A549 lung cells. Biomedical and Environmental Sciences, 24, 593-601.

[29] Harvilchuck, J.A., Pu, X., Klaunig, J.E. and Carlson, G.P. (2009) Indicators of oxidative stress and apoptosis in mouse whole lung and Clara cells following exposure to styrene and its metabolites. Toxicology, 264, 171-178. doi:10.1016/j.tox.2009.08.001

[30] Meszka-Jordan, A., Mahlapuu, R., Soomets, U. and Carlson, G. P. (2009) Oxidative stress due to (R)-styrene oxide exposure and the role of antioxidants in non-Swiss albino (NSA) mice. Journal of Toxicology and Environmental Health, Part A, 72, 642-650. doi:10.1080/15287390902769436

[31] Mörbt, N., Tomm, J., Feltens, R., Mögel, I., Kalkhof, S., Murugesan, K., Wirth, H., Vogt, C., Binder, H., Lehmann, I. and von Bergen, M. (2011) Chlorinated benzenes cause concomitantly oxidative stress and induction of apoptotic markers in lung epithelial cells (A549) at non-acute toxic concentrations. Journal of Proteome Research, 10, 363378. doi:10.1021/pr1005718

[32] Gould, N.S., White, C.W. and Day, B.J. (2009) A role for mitochondrial oxidative stress in sulfur mustard analog 2-chloroethyl ethyl sulfide-induced lung injury and antioxidant protection. Journal of Pharmacology and Experimental Therapeutics, 328, 732-739. doi:10.1124/jpet.108.145037

[33] Choi, Y.H. and Park, H.S. (2012) Apoptosis induction of U937 human leukemia cells by diallyl trisulfide induces through generation of reactive oxygen species. Journal of Biomedical Science, 19, 50. doi:10.1186/1423-0127-19-50

[34] Pylkkänen, L., Stockmann-Javala, H., Alenius, H., Husgafvel-Pursiainen, K. and Savolainen, K. (2009) Wood dusts induce the production of reactive oxygen species and caspase- 3 activity in human bronchial epithelial cells. Toxicology, 262, 265-270. doi:10.1016/j.tox.2009.06.019

[35] Kovacic, P. and Somanathan, R. (2010) Dermal toxicity and environmental contamination: Electron transfer, reactive oxygen species, oxidative stress, cell signaling, and protection by antioxidants. Reviews of Environmental Contamination and Toxicology, 203, 119-138. doi:10.1007/978-1-4419-1352-4_4

[36] Tewari-Singh, N., Agarwal, C., Huang, J., Day, B.J., White, C.W. and Agarwal, R. (2011) Efficacy of glutathione in ameliorating sulfur mustard analog-induced toxicity in cultutred skin epidermal cells and in SKH-1 mouse skin in vivo. Journal of Pharmacology and Experimental Therapeutics, 336, 450-459. doi:10.1124/jpet.110.173708

[37] Tewari-Singh, N, Gu, M., Ahotriya, S., Gomnez, J., Agarwal, C., White, C.W. and Agarwal, R. (2011) Mechanisms of sulfur mustard analog 2-chloeroethyl ethyl sulfide-induced DNA damage in skin epidermal cells and fibroblasts. Free Radical Biology \& Medicine, 51, 22722280. doi:10.1016/j.freeradbiomed.2011.08.020

[38] Pal, A., Twari-Singh, N., Gu, M., Agarwal, C., Huang, J., 
Day, B. J., White, C. W. and Agarwal, R. (2009) Sulfur mustard analog induces oxidative stress and activates signaling cascades in the skin of SKH-1 hairless mice. Free Radical Biology \& Medicine, 47, 1640-1651. doi:10.1016/i.freeradbiomed.2009.09.011

[39] Son, Y.-O., Hitron, J.A., Wang, X., Chang, Q., Pan, J., Zhang, Z., Liu, J., Wang, S., Lee, J.-C. and Shi, X. (2010) $\mathrm{Cr}(\mathrm{IV})$ induces mitochondrial-mediated and caspase-dependent apoptosis through reactive oxygen species-mediated p53 activation in JB6 C141 cells. Toxicology and Applied Pharmacology, 245, 226-235. doi:10.1016/j.taap.2010.03.004

[40] Murray, A.R., Kisin, E., Leonard, S.S., Young, S.H., Kommineni, C., Kagan, V.E., Castranova, V. and Shvedova, A.A. (2009) Oxidative stress and inflammatory response in dermal toxicity of single walled carbon nanotubes. Toxicology, 257, 161-171. doi:10.1016/j.tox.2008.12.023

[41] Nabeshi, H., Yoshikawa, T., Matsuyama, K., Nakazato, Y., Tochigi,S., Kondoh, S., Hirai, T., Akase, T., Nagano, K., Abe, Y., Yoshioka, Y., Kamada, H., Itoh, N., Tsunoda, S.-I, and Tsutsumi, Y. (2011) Amorphous nanosilica induce endocytosis-dependent ROS generation and DNA damage in human keratinocytes. Particle and Fibre Toxicology, 8, 1. doi:10.1186/1743-8977-8-1

[42] Daraei, B., Pourahmad, J., Hamidi-Pour, N., Hosseneini, M.-J., Shaki, F. and Solleimani, M. (2012) Uranyl acetate induces oxidative stress and mitochondrial membrane potential collapse in the human dermal fibroblast primary cells. Iranian Pharmaceutical Research, 11, 495-501.

[43] Terao, J., Minami, Y. and Bando, N. (2011) Singlet molecular oxygen-quenching activity of carotenoids: Relevance to protection of the skin from photoaging. Journal of Clinical Biochemistry and Nutrition, 48, 57-62. doi:10.3164/jcbn.11-008FR

[44] White, P.O., Tribout, H. and Baron, E. (2012) Protective mechanisms of green tea polyphenols in skin. Oxidative Medicine and Cellular Longevity, 2012, Article ID: 560682, 8 Pages. doi:10.1155/2012/560682

[45] Ascenso, A., Ribeiro, H.M., Marques, H.C. and Simoes, S. (2011) Topical delivery of antioxidants. Current Drug Delivery, 8, 640-660. doi:10.2174/156720111797635487

[46] Ozbek, E. (2012) Induction of oxidative stress in kidney. International Journal of Nephrology, 2012, Article ID: 465897, 9 Pages. doi:10.1155/2012/465897

[47] Ramatillah, D.L., Gillani, S.W. and Suardi, M. (2012) Effect of cytotoxic medications (MTX, cisplatin, 5-FU and cyclophosphamide) against creatinine clearance. Patient relationships and creatinine clearance urea with cancer patients. International Journal of Pharmacy Education, 3, 240-244.

[48] Deavall, D.G., Mertin, E.A., Horner, J.M. and Roberts, R. (2012) Drug-induced oxidative stress and toxicity. Journal of Toxicology, 2012, Article ID: 645460, 13 Pages. doi:10.1155/2012/645460

[49] Yao, X., Panichpisal, K., Kurtzman, N. and Nugent, K. (2007) Cisplatin nephrotoxicity; a review. The American Journal of the Medical Sciences, 334, 115-124. doi:10.1097/MAJ.0b013e31812dfe1e
[50] Pourahmad, J., Hosseini M.J., Eskandari, M.R., Shekarabi, S.M. and Daraei, B. (2010) Mitochondrial/lysosomal toxic cross-talk plays a key role in cisplatin nephrotoxicity. Xenobiotica, 40, 763-771. doi:10.3109/00498254.2010.512093

[51] Li, Y., Li, X., Wong, Y.S., Chen, T., Zhang, H., Liu, C. and Zheng, W. (2011) The reversal of cisplatin-induced nephrotoxicity by selenium nanoparticles functionalized with 11-mercapto-1-undecanol by inhibition of ROSmediated apoptosis. Biomaterials, 32, 9068-9076. doi:10.1016/j.biomaterials.2011.08.001

[52] El-Sayed, E.-S.M., Abd-Ellah, M.F. and Attia, S.Y.M. (2008) Protective effect of captopril against cisplatin-induced nephrotoxicity in rats. Pakistan Journal of Pharmaceutical Sciences, 21, 255-261.

[53] Pan, H., Mukhopadhyay, P., Rajesh, M., Patel, V., Mukhopadhyay, B., Gao, B., Kasko, G. and Pacher, P. (2009) Cannabidol attenuates cisplatin-induced nephrotoxicity by decreasing oxidative/nitrosative stress, inflammation, and cell death. Journal of Pharmacology and Experimental Therapeutics, 328, 708-714. doi:10.1124/jpet.108.147181

[54] Denamur, S., Tyteca, D., Marchand-Brynaert, J., Van Bambeke, F., Tulkens, P. M. Courtoy, P. J. and MingeotLeclercq, M-P. (2011) Role of oxidative stress in lysosomal membrane permeabilization and apoptosis induced by gentamicin, an aminoglycoside antibiotic. Free Radical Biology \& Medicine, 51, 1656-1665. doi:10.1016/j.freeradbiomed.2011.07.015

[55] Randjelovic, P., Veljkovic, S., Stojiljkovic, N., Jankovic-Velickovic, L., Sokolovic, D., Stoiljkovic, M. and Ilic, I. (2012) Salicylic acid attenuates gentamicin-induced nephrotoxicity in rats. The Scientific World Journal, 2012, Article ID: 390613, 6 Pages. doi: $10.1100 / 2012 / 390613$

[56] Hanly, L., Chen, N., Rieder, M. and Koren, G. (2009) Ifosfamide nephrotoxicity in children: A mechanistic base for pharmacological prevention. Expert Opinion on Drug Safety, 8, 155-168. doi: $10.1517 / 14740330902808169$

[57] O'Connell, S., Tuite, N., Slattery, C., Ryan, M.P. and McMorrow, T. (2012) Cyclosporin A-induced oxidative stress in human renal mesangial cells; a role for ERK 1/2 MAPK signaling. Toxicological Sciences, 126, 101113. doi:10.1093/toxsci/kfr330

[58] Durante, P., Romero, F., Pérez, M., Chávez, M. and Parra, G. (2010) Effect of uric acid on nephrotoxicity induced by mercuric chloride in rats. Toxicology and Industrial Health, 26, 163-174. doi:10.1177/0748233710362377

[59] Tarasub, N., Tarasub, C. and Ayutthaya, W.D.N. (2011) Protective role of curcumin on cadmium-induced nephrotoxicity in rats. Journal of Environmental Chemistry and Ecotoxicology, 3, 17-24.

[60] Yousef, J., Chen, G., Hill, P.A., Nation, R. L. and Li, J. (2011) Melatonin attenuates colistin-induced nephrotoxicity in rats. Antimicrobial Agents and Chemotherapy, 55, 4044-4049. doi:10.1128/AAC.00328-11

[61] Pujalté, I., Passagne, I., Brouillaud, B.L.,Tréguer, M., 
Durand, E., Ohayon-Courtés, C. and L'Azou, B. (2011) Cytotoxicity and oxidative stress induced by different metallic nanoparticles on human kidney cells. Particle and Fibre Toxicology, 8, 10. doi:10.1186/1743-8977-8-10

[62] Shafik, A., Khodeir, M.M. and Fadel, M.S. (2011) Animal study of anthracycline-induced cardiotoxicity and nephrotoxicity and evaluation of protective agents. Journal of Cancer Science \& Therapy, 3, 96-103.

[63] Shi, R., Huang, C.-C., Aronstam, R.S., Ercal, N., Martin, A. and Huang, Y.-W. (2009) N-acetylcysteine amide decreases oxidative stress but not cell death induced by doxorubicin in H9c2 cardiomyocytes. BMC Pharmacology, 9, 7. doi:10.1186/1471-2210-9-7

[64] Aluise, C.D., St. Clair, D., Vore, M. and Butterfield, D.A. (2009) In vivo amelioration of adrinamycin induced oxidative stress in plasma by gamma-glutamylcysteine ethyl ester (GCEE). Cancer Letters, 282, 25-29. doi:10.1016/j.canlet.2009.02.047

[65] Sawyer, D.B., Peng, X., Chen, B., Pentassuglia, L. and Lim, C.C. (2010) Mechanisms of anthracycline cardiac injury: Can we identify strategies for cardio-protection? Progress in Cardiovascular Diseases, 53, 105-113. doi:10.1016/j.pcad.2010.06.007

[66] Ferreira, A.L.A., Matsubara, L.S. andMatsubara, B.B. (2008) Anthracycline-induced cardiotoxicity. Cardiovascular \& Hematological Agents in Medicinal Chemistry, 6, 278-281. doi:10.2174/187152508785909474

[67] Vergely, C., Delemasure, S., Cottin, Y. and Rochette, L. (2007) Preventing the cardiotoxic effects of anthracyclines: From basic concepts to clinical data. Heart and Metabolism, 35, 1-7.

[68] James, H.D. (2012) Dexrazane for the prevention of cardiac toxicity and treatment of extravasation injury from the anthracycline antibiotic. Current Pharmaceutical Biotechnology, 13, 1949-1956. doi:10.2174/138920112802273245

[69] Ferroni, P., Della-Morte, D., Palmirotta, R., McClendon, M., Testa, G., Abete, P., Rengo, F., Rundek, T., Guadagni, F. and Roselli, M. (2011) Platinum-based compounds and risk for cardiovascular toxicity in the elderly: Role of the antioxidants in chemoprevention. Rejuvenation Research, 14, 293-308. doi:10.1089/rej.2010.1141

[70] Cheng, C.F., Juan, S.H., Chen, J.J., Chao, Y.C., Chen, H.H., Lian, W.S., Lu, C.Y., Chang, C.I., Chiu, T.H. and Lin, H. (2008) Pravatatin attenuates carboplatin-induced cardiotoxicity via inhibition of oxidative stress associated apoptosis. Apoptosis, 13, 883-894. doi:10.1007/s10495-008-0214-9

[71] Müller-Krebs, S., Kihm, L.P., Zeier, B., Gross, M.L., Wieslander, A., Haug, U., Zeier, M. and Schwenger, V. (2010) Glucose degradation products result in cardiovascular toxicity in a rat model of renal failure. Peritoneal Dialysis International, 30, 35-40. doi:10.3747/pdi.2009.00031

[72] Himmele, R., Sawin, D.-A. and Diaz-Buxo, J.A. (2011) GDPs and AGEs: Impact on cardiovascular toxicity in dialysis patients. Advances in Peritoneal Dialysis, 27, 2226.

[73] Kovacic, P. and Somanathan, R. (2011) Cell signaling and receptors in toxicity of advanced glycation products (AGEs): $\alpha$-dicarbonyl, radicals, oxidative stress and antioxidants. Journal of Receptors and Signal Transduction Research, 31, 332-339. doi:10.3109/10799893.2011.607171

[74] Fan, L., Sawbridge, D., George, V., Teng, L., Baily, A., Kitchen, I. and Li, J.-M. (2009) Chronic cocaine-induced cardiac oxidative stress and mitogen-activated protein kinase activation: The role of Nox 2 oxidase. Journal of Pharmacology and Experimental Therapeutics, 328, 99106. doi:10.1124/jpet.108.145201

[75] LeBlanc, A.J., Mosely, A.M., Chen, B.T., Frazer, D., Castranova, V. and Nurkiewicz, T.R. (2010) Nanoparticle inhalation impairs coronary microvascular reactivity via a local reactive oxygen species-dependent mechanism. Cardiovascular Toxicology, 10, 27-36. doi:10.1007/s12012-009-9060-4

[76] Kin, J.B., Kim, C., Choi, E., Park, H., Pak, H.N., Lee, M. H., Shin, D.C., Hwang, K.C. and Joung, B. (2012) Particulate air pollution induces arrhythmia via oxidative stress and calcium calmodulin kinase II activation. Toxicology and Applied Pharmacology, 259, 66-73. doi:10.1016/j.taap.2011.12.007

[77] Alissa, E. and Ferns, G.A. (2011) Heavy metal poisoning and cardiovascular disease. Journal of Toxicology, 2011, Article ID: 870125, 21 Pages. doi:10.1155/2011/870125

[78] Azevedo, B.F., Furieri, L.B., Peçanha, F.M., Wiggers, G.A., Vassallo, P.F., Simõexs, M.R., Fiorim, J., de Batista, P., Fioresi, M., Rossoni, L., Stefanon, I., Alonso, M.J., Salaices, M. and Vassallo, D.V. (2012) Toxic effects of mercury on the cardiovascular and central nervous systems. Journal of Biomedicine and Biotechnology, 2012, Article ID: 949048. doi:10.1155/2012/949048

[79] Kovacic, P and Jacintho, J.D. (2001) Reproductive toxins: pervasive theme of oxidative stress and electron transfer. Current Medicinal Chemistry, 8, 863-892. doi:10.2174/0929867013372878

[80] Kovacic, P and Somanathan, R. (2006) Mechanism of teratogenesis: Electron transfer, reactive oxygen species and antioxidants. Birth Defects Research Part C, 78, 308325. doi:10.1002/bdrc. 20081

[81] Hansen, J.M. (2006) Oxidative stress as a mechanism of teratogenesis. Birth Defects Research Part C, 78, 293-307. doi: $10.1002 / \mathrm{bdrc} .20085$

[82] Oyagbemi, A.A., Adedara, I.A., Saba, A.B. and Farombi, E.O. (2010) Role of oxidative stress in reproductive toxicity induced by coadministration of chloroamphenicol and multivitamin-haematinics complex in rats. Basic \& Clinical Pharmacology \& Toxicology, 107, 103-108. doi:10.1111/j.1742-7843.2010.00561.x

[83] Ihsan, A., Wang, X., Liu, Z., Wang, Y., Huang, X., Liu, Y., Yu, H., Zhang, H., Li, T., Yang, C. and Yuan, Z. (2011) Long-term mequindox treatment induced endocrine and reproductive toxicity via oxidative stress in male Wistar rats. Toxicology and Applied Pharmacology, 252, 281288. doi:10.1016/j.taap.2011.02.020

[84] Wang, N., Qian, H.Y., Zhou, X.Q., Li, X.Q. and Sun, Z.W. (2012) Mitochondrial energy metaboilism dysfunction involved in reproductive toxicity of mice caused by en- 
dosulfan and protective effects of vitamin E. Ecotoxicology and Environmental Safety, 82, 96-103. doi:10.1016/i.ecoenv.2012.05.014

[85] Amin, A., Abraham, C., Hamza, A.A., Abdalla, Z.A., Al-Shamsi, S.B., Harethi, S.S. and Daoud, S. (2012) A standardized extract of Ginkgo biloba neutralizes cisplatin-mediated reproductive toxicity in rats. Journal of Biomedicine and Biotechnology, 2012, Article ID: 362049, 11 Pages. doi:10.1155/2012/362049

[86] Ait H.N., Slimani, M., Merad-Boudia, B. and Zaoui, C. (2009) Reproductive toxicity of lead acetate in adult male rats. American Journal of Veterinary Research, 38-50.

[87] Subramanian, S., Rajendiran, G., Skhar, P., Gowri, C., Govindarajulu, P. and Aruldhas, M.M. (2006) Reproductive toxicity of chromium in adult bonnet monkeys (Macaca radiate Geoffrey) Reversible oxidative stress in the seman. Toxicology and Applied Pharmacology, 215, 237-249. doi:10.1016/j.taap.2006.03.004

[88] Hari, P. and Sreenivasula R.P. (2012) Effect of restraint stress on lead-induced male reproductive toxicity in rats. Journal of Experimental Zoology, 317A, 455-465. doi:10.1002/jez.1738

[89] Kaushal, N. and Bansal, M.P. (2009) Diminished reproductive potential of male mice in response to selenium-induced oxidative stress: Involvement of HSP70, HSP70-2, and MSJ-1. Journal of Biochemical and Molecular Toxicology, 23, 125-136. doi:10.1002/jbt.20276

[90] Borniquel, S., Valle, I., Cadenas, S., Lamas, S. and Monsalve, M. (2006) Nitric oxide regulates mitochondrial oxidative stress protection via the transcriptional coactivator PGC-1 $\alpha$. The FASEB Journal, 20, E1216-E1227. doi:10.1096/fj.05-5189fje

[91] Chen, C.-H., Sun, L. and Mochly-Rosen, D. (2010) Mitochondrial aldehyde and dehydrogenase and cardiac diseases. Cardiovascular Research, 88, 51-57. doi:10.1093/cvr/cvq192

[92] Wang, J., Wang H., Hao, P., Xue, L., Wei, S., Zhang, Y. and Chen, Y. (2011) Inhibition of aldehyde dehydrogenase 2 by oxidative stress is associated with cardiac dysfunction in diabetic rats. Molecular Medicine, 17, 172-179.

[93] Mariappan, N., Soorappan, R.N., Haque, M., Siramula, S. and Francis, J. (2007) TNF- $\alpha$-induced mitochondrial oxidative stress and cardiac dysfunction: Restoration by superoxide dismutase mimetic Tempol. American Journal of Physiology-Heart and Circulatory, 293, H2726-H2737. doi:10.1152/ajpheart.00376.2007

[94] Montaigne, D., Hurt, C. and Neviere, R. (2012) Mitiochnodria death/survival signaling pathways in cardiotoxicity induced by anthracyclines and anticancer-targeted therapies. Biochemistry Research International, 2012, Article ID: 951539, 12 Pages. doi:10.1155/2012/951539

[95] Victor, V.M., Apostolova, N., Herance, R., HernandezMijares, A. and Rocha, M. (2009) Oxidative stress and mitochondrial dysfunction in atherosclerosis: Mitochondria-targeted anitoxidants as potential therapy. Current Medicinal Chemistry, 16, 4654-4667. doi:10.2174/092986709789878265

[96] Aliev, G., Palacios, H.H., Gasimov, E., Oberenovich, M.E., Morales, L., Leszek, J., Bragin, V., Herrera, A.S. and Gokhman, D. (2010) Oxidative stress induced mitocondrial failure and vascular hyperfusion as key initiator for the development of Alzheimer's disease. Pharmaceuticals, 3, 158-187. doi:10.3390/ph3010158

[97] Ferreiro, E., Balseiras, I., Ferreira, I.L., Costa, R.O., Rego, A.C., Pereira, C.F. and Oiliveira, C.R. (2012) Mitochondrial- and endoplasmic reticulum-associated oxidative stress in Alzheimer's disease: From pathogenesis to biomarkers. International Journal of Cell Biology, 2012, Article ID: 735206, 23 Pages. doi: $10.1155 / 2012 / 735206$

[98] Cui, H., Kong, Y. and Zhang, H. (2012) Oxidative stress, mitochondrial dysfunction, and aging. Journal of Signal Transduction, 2012, Article ID: 646354, 13 Pages. doi: $10.1155 / 2012 / 646354$

[99] Lee, H.-C. and Wei, Y.-H. (2007) Oxidative stress, mitochondrial DNA mutation, and apoptosis in aging. Experimental Biology and Medicine, 232, 592-606.

\section{ABREVIATIONS}

OS, oxidative stress;

ROS, reactive oxygen species;

RNS, reactive nitrogen species;

ET, electron transfer;

AO, antioxidant;

COPD, chronic obstructive pulmonary disease;

GABA, gamma amino butyric acid;
HSP, heat shock proteins;

LPP, lipid peroxidation products;

CIS, cisplatin;

SOD, superoxide dismutase;

GSH, glutathione;

8-OHdG, 8-hydroxydeoxyguanosine;

MDA, malondialdehyde;

MEQ, Mequindox. 